\title{
Magnetism of substitutional Co impurities in graphene: Realization of single $\pi$ vacancies
}

\author{
E. J. G. Santos, ${ }^{*}$ D. Sánchez-Portal,$^{\dagger}$ and A. Ayuela ${ }^{\ddagger}$ \\ Centro de Física de Materiales (CSIC-UPV/EHU)-Materials Physics Center (MPC), Apartado 1072, 20080 San Sebastián, Spain \\ and Donostia International Physics Center (DIPC), Paseo Manuel de Lardizabal 4, 20018 San Sebastián, Spain
}

(Received 11 December 2009; revised manuscript received 19 February 2010; published 26 March 2010)

\begin{abstract}
We report ab initio calculations of the structural, electronic, and magnetic properties of a graphene monolayer substitutionally doped with $\mathrm{Co}\left(\mathrm{Co}_{\text {sub }}\right)$ atoms. These calculations are done within density-functional theory using the generalized gradient approximation. We focus in Co because among traditional ferromagnetic elements $\left(\mathrm{Fe}, \mathrm{Co}\right.$, and $\mathrm{Ni}$ ), only $\mathrm{Co}_{\text {sub }}$ atoms induce spin polarization in graphene. Our results show the complex magnetism of Co substitutional impurities in graphene, which is mapped into simple models such as the $\pi$-vacancy and Heisenberg model. The links established in our work can be used to bring into contact the engineering of nanostructures with the results of $\pi$ models in defective graphene. In principle, the structures considered here can be fabricated using electron irradiation to create defects and depositing Co at the same time.
\end{abstract}

DOI: 10.1103/PhysRevB.81.125433

PACS number(s): 73.90.+f, 75.70.-i, 61.48.De

\section{INTRODUCTION}

The peculiar electronic and magnetic properties of graphene monolayers have recently attracted much attention. ${ }^{1-4}$ New types of electronic and, particularly, spintronic devices based on graphene have been proposed. These graphene structures also drive an increasing interest to study defects, which are always expected in real-life devices. Intrinsic defects have been already widely studied ${ }^{4-9}$ and extrinsic defects, such as substitutional atoms, are presently under intense research. ${ }^{10-13}$ In spite of the growing interest about substitutional defects in graphene, little is known on magnetic properties such as spin polarizations and couplings. Recent experiments show that metal atoms can be incorporated to graphene. Gan et al. ${ }^{14}$ using high-resolution transmission electron microscopy (HRTEM) have observed substitutional $\mathrm{Au}$ and $\mathrm{Pt}$ atoms in graphene monolayers. They observed large activation energies for in-plane migration, which point toward strong covalent carbon-metal bonding and high stability for such substitutional defects. The doping of graphene with transition metals and their spintronics applications is underway. Thus, the study of magnetic substitutional impurities is of importance for both, the theoretical understanding of magnetism for atomic defects in graphene and the explanation of experiments in progress that dope graphene with elements of the traditional magnetic bulks (Fe, $\mathrm{Co}$, and $\mathrm{Ni}$ ).

In this work, we present a study of the structural, electronic, and magnetic properties of substitutional $\mathrm{Co}\left(\mathrm{Co}_{\text {sub }}\right)$ atoms in a graphene monolayer. We show that Co has spin polarization. This is in contrast to $\mathrm{Fe}$ and $\mathrm{Ni}$ substitutions which surprisingly do not show spin polarization. ${ }^{13,15} \mathrm{We}$ observe that there is a one-to-one correspondence between the expected behavior for single vacancies in a simple $\pi$-tight-binding model of graphene and that found for the $\mathrm{Co}_{\text {sub }}$ defects. The Co atom stabilizes a symmetric structure of the carbon vacancy. The electronic structure of the $\mathrm{Co}_{\text {sub }}$ defect at the Fermi energy $\left(\mathrm{E}_{\text {Fermi }}\right)$ is dominated by a single level with a strong contribution from the $p_{z}$ orbitals of the neighboring $\mathrm{C}$ atoms. Each $\mathrm{Co}_{\text {sub }}$ defect shows a spin mo- ment of $1.0 \mu_{B}$. The total spin, however, follows closely Lieb's theorem for bipartite lattices ${ }^{16}$ and depends on the number of Co substitutions in each sublattice. Thus, magnetic couplings are predominantly (anti)ferromagnetic for $\mathrm{Co}_{\text {sub }}$ defects sited in the (opposite) same sublattice. Our calculations also show the dependence of the couplings on the crystalline direction and relative position of the defects.

\section{COMPUTATIONAL DETAILS}

We have used the SIESTA code ${ }^{17,18}$ for our calculations. Within density-functional theory we have used the generalized gradient approximation GGA for the exchangecorrelation potential. We have checked that the normconserving pseudopotential ${ }^{19}$ used for Co satisfactorily reproduces the band structure and spin moment of bulk Co. This pseudopotential includes nonlinear core corrections ${ }^{20}$ for exchange and correlation, with a pseudocore matching radius of 0.67 a.u. Computational parameters guarantee the convergence of relative energies for different magnetic configurations within the meV. ${ }^{13}$ The results obtained with VASP code, ${ }^{21,22}$ using a well converged plane-wave cutoff of 400 $\mathrm{eV}$ and the projected-augmented-wave scheme, are almost identical to those obtained with SIESTA.

\section{RESULTS}

In this section, we shall describe after bonding and geometrical comments the band structure and magnetism of substitutional Co impurities periodically distributed in graphene. We are calculating substitutional Co atoms in $4 \times 4$ and 8 $\times 8$ supercells with concentration ranging between $3 \%$ and $0.8 \%$, respectively. We are not dealing with isolated Co impurities in graphene in the limit of infinite dilution because the required techniques are different from those used in this work. Let us consider the bonding and band structure in more details for one and two Co substitutionals in graphene supercells. 

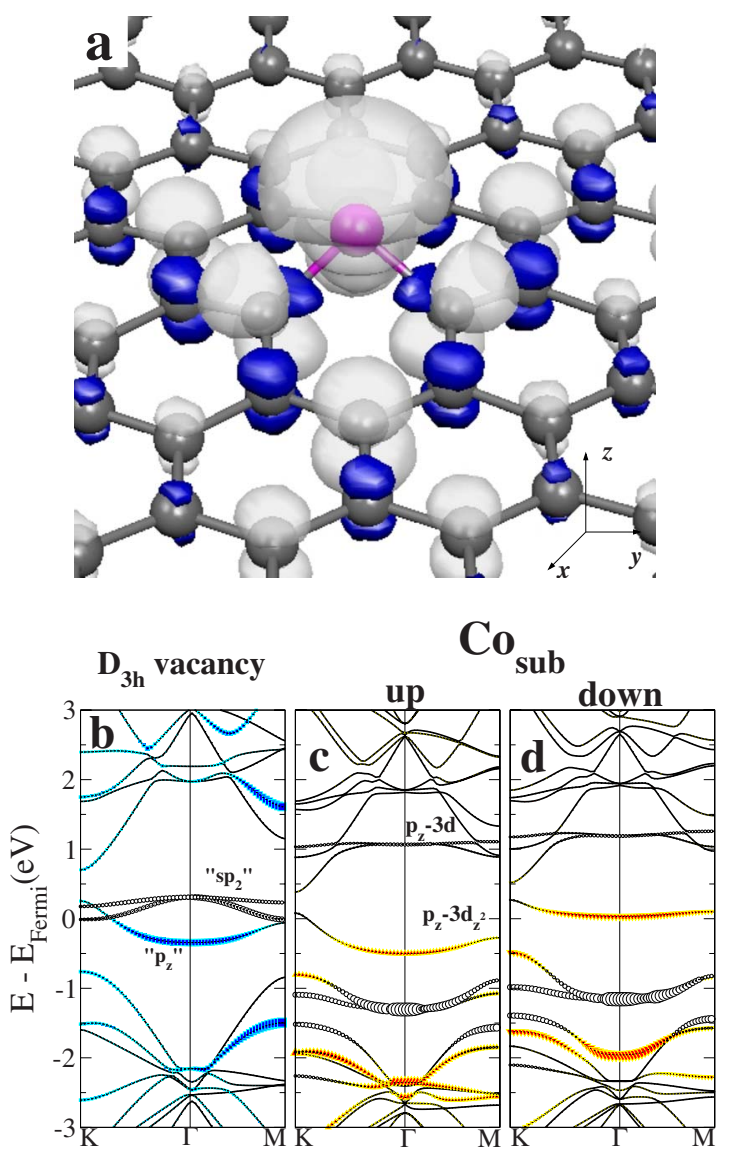

FIG. 1. (Color online) (a) Isosurface of the spin density induced by a $\mathrm{Co}_{s u b}$ defect. Positive and negative spin densities correspond to light and dark surfaces with isovalues of $\pm 0.008 \mathrm{e}^{-} / \mathrm{Bohr}^{3}$, respectively. Panel (b) presents the spin-unpolarized band structure of an unreconstructed $D_{3 h}$ carbon vacancy. Panel (c) and (d) show, respectively, the majority and minority spin band structure a $\mathrm{Co}_{\text {sub }}$ defect in a similar cell. The size of filled symbols in panel (b) indicates the contribution of the $p_{z}$ orbitals of the $\mathrm{C}$ atoms surrounding the vacancy, whereas empty symbols correspond to the $s p^{2}$ character. In panels (c) and (d), the size of filled and empty circles denote the contribution of hybridized Co $3 d_{z^{2}} \mathrm{C} 2 p_{z}$ and Co $3 d-\mathrm{C}$ $2 s p^{2}$ characters, respectively. $\mathrm{E}_{F}$ is set to zero.

\section{A. Geometrical parameters and binding energies}

The relaxed geometry and the magnetization density for a $\mathrm{Co}_{\text {sub }}$ defect in a graphene monolayer is given in Fig. 1(a). The Co atom appears displaced $0.92 \AA$ from the carbon surface, and it has a symmetrical threefold coordinated structure with Co-C distances of $1.77 \AA$. The three C-Co-C bond angles are quite similar and have a value $\sim 97^{\circ}$. The binding energy of the Co atom to the carbon vacancy in graphene is $7.6 \mathrm{eV}$. Co has the highest binding energy to the reconstructed vacancy among three late transition metals. This high stability of $\mathrm{Co}_{s u b}$ is madespecial when thinking in future experimental realizations of ordered arrays distributing ordered substitutional metals in graphene. In comparison, the most stable adsorption configuration of $\mathrm{Co}$ on graphene has a much lower energy of $1.3 \mathrm{eV}$. Therefore, $\mathrm{Co}_{\text {sub }}$ defects are likely to be found in carbon nanostructures synthesized using Co-containing catalysts, as seems to be the case for $\mathrm{Ni}^{23}$
Furthermore one could expect that $\mathrm{Co}_{\text {sub }}$ defects can be fabricated in a controlled way by creating vacancies in graphene and subsequently depositing Co. The presence of $\mathrm{Co}_{s u b}$ defects could be detected today by scanning tunneling microscopy (STM), ${ }^{24}$ X-ray adsorption techniques, ${ }^{23}$ and HRTEM. ${ }^{14}$

\section{B. Band structures and spin densities: mapping to $\pi$ vacancies}

Figure 1(a) shows the spin polarization pattern induced by the presence of a $\mathrm{Co}_{s u b}$ impurity. The spin polarization induced in the neighboring carbon atoms has a clear $p_{z}$-like shape. The sign of the spin polarization follows the bipartite character of graphene: the polarization aligns parallel (antiparallel) to the spin moment of the Co impurity for carbon atoms sitting in the opposite (same) sublattice than the Co atom. The total spin moment is $1.0 \mu_{B}$. Using Mulliken population analysis, $0.44 \mu_{B}$ are assigned to the Co atom; $0.20 \mu_{B}$, to the three first carbon neighbors; and $0.36 \mu_{B}$, delocalized in the rest of the layer. The spin polarization decays with distance very slowly. Both Co and carbon orbitals contribute, and this points also to the strong $\mathrm{Co}-\mathrm{C}$ hybridization.

To understand the origin of this spin polarization, we now analyze in detail the band structure of this system. Figures 1(c) and 1(d) present the results for a $\mathrm{Co}_{\text {sub }}$ defect in a 4 $\times 4$ graphene supercell. Similar results are obtained using an $8 \times 8$ cell. For comparison, the panel (b) shows the spin compensated band structure of a single unreconstructed carbon vacancy, the so called $D_{3 h}$ vacancy. ${ }^{6}$ For the $D_{3 h}$ vacancy, three defect states appear in a range of $\sim 0.7 \mathrm{eV}$ around the Fermi energy $\left(\mathrm{E}_{F}\right)$. Two states appearing at $0.3 \mathrm{eV}$ above $\mathrm{E}_{F}$ at $\Gamma$ have a large contribution from the $s p^{2}$ lobes of the $\mathrm{C}$ atoms surrounding the vacancy. Other state at $0.35 \mathrm{eV}$ below $\mathrm{E}_{F}$ at $\Gamma$ shows a predominant $p_{z}$ contribution. The level corresponds with the defect state that appears at $\mathrm{E}_{F}$ for a vacancy in a simplified $\pi$ tight-binding description of graphene.

When a Co atom is bound to the vacancy, the defect states described above hybridize with the $3 d$ states of Co. The two $2 s p^{2}$ defect bands, now an antibonding combination of Co $3 d$ and the original C $2 s p^{2}$ vacancy levels, are pushed at higher energies, $\sim 1.0 \mathrm{eV}$ above $\mathrm{E}_{F}$ [see Figs. 1(c) and 1(d)]. The singly-occupied $p_{z}$ state, now hybridized mainly with the Co $3 d_{z^{2}}$ orbital, remains at the $\mathrm{E}_{F}$ and becomes almost fully spin polarized. Thus, the $\mathrm{Co}_{s u b}$ impurity becomes analogous to a vacancy in the $\pi$ tight-binding model of graphene. This $p_{z} \mathrm{C}$ band at the Fermi energy brings our results concerning magnetic moments into contact with the Lieb s theorem, as shown in the following section for several Co atoms in the cell.

The splitting between majority and minority $p_{z}$ defect bands is $\sim 0.50 \mathrm{eV}$. The spin splitting is much smaller for all other bands. Considering other transitions metals close to $\mathrm{Co}$ and important in catalysis, $\mathrm{Ni}$ has the $p_{z}$ band fully occupied while for $\mathrm{Fe}$ it is empty. As a consequence $\mathrm{Fe}_{\text {sub }}$ and $\mathrm{Ni}_{\text {sub }}$ do not show spin polarization. ${ }^{25}$

Next we consider whether the presence of the $\mathrm{C} p_{z}$ band induced by Co depends on supercell size. The electronic structure for a $8 \times 8$ supercell show such band, as already 


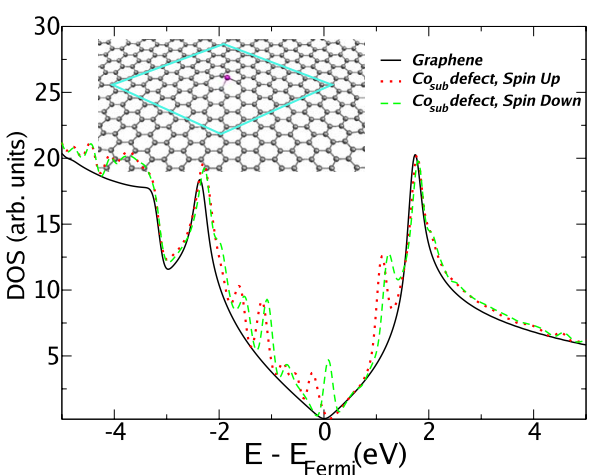

FIG. 2. (Color online) DOS around the Fermi energy for graphene and $\mathrm{Co}_{\text {sub }}$ in $8 \times 8$ supercell. DOS for graphene is given with full line (black). $\mathrm{Co}_{\text {sub }}$ DOS is splitted in up with dark gray (red) and down with light gray (green). Note that the $\mathrm{Co}_{\text {sub }}$ DOS is superimposed on graphene cone and it changes mainly at its center.

seen in the $4 \times 4$ case. To avoid a spaghetti picture, we show here the density of states (DOS) with a broadening of 0.12 $\mathrm{eV}$ for the larger $8 \times 8$ cell in Fig. 2 . Around Fermi energy, the spin splitted DOS structure for the $8 \times 8$ cell follows the band trends previously discussed for a smaller cell. The destruction of Dirac cone along high-symmetry directions has also been observed in other papers with unreconstructed vacancies. ${ }^{6}$ When comparing with pristine graphene, the total DOS farther from the Fermi energy goes up with strong oscillations and increases with reminiscences of the Dirac spectrum in graphene.

From Fig. 2 we also learn that the Fermi energy remains almost the same for the $8 \times 8$ supercell of $\mathrm{Co}_{\text {sub }}$ and pristine graphene. This is indicative of negligible charge transfer from Co to graphene. We have explicitly checked this charge neutrality for the large $8 \times 8$ supercell by looking at the Mulliken population around each $\mathrm{C}$ or $\mathrm{Co}$ atom. We observe that the $\mathrm{Co}$ atom remains almost neutral below 0.06e independently of the used cell $4 \times 4$ or $8 \times 8$. The calculation indicates that the C-Co bonding is covalent. This charge neutrality in the Co substitutional positions is generally different from metal atoms attached externally to graphenic nanostructures. ${ }^{26}$

The magnetic moment appears in Co atoms and in the nearest-neighbors carbon atoms of graphene lattice [Fig. 1(a)]. The $\mathrm{C}$ spin polarization is not negligible and it is similar to the unreconstructed vacancy. The carbon spin magnetization implies that this picture is very similar to the setting of the Lieb theorem, where spin polarization is an intrinsic property of the defective bipartite lattice. However Lieb theorem is global referring to the total system magnetization and does not enters into the local description of the magnetic interaction, which is going to be described in the following section taking into account several $\mathrm{Co}_{\text {sub }}$ defects.

\section{C. $A A$ and $A B$ magnetic couplings: Lieb theorem for the total magnetization}

We consider next the magnetic couplings between $\mathrm{Co}_{\text {sub }}$ defects in a large $8 \times 8$ supercell with two $\mathrm{Co}_{\text {sub }}$ impurities. We calculate the energy difference between different spin
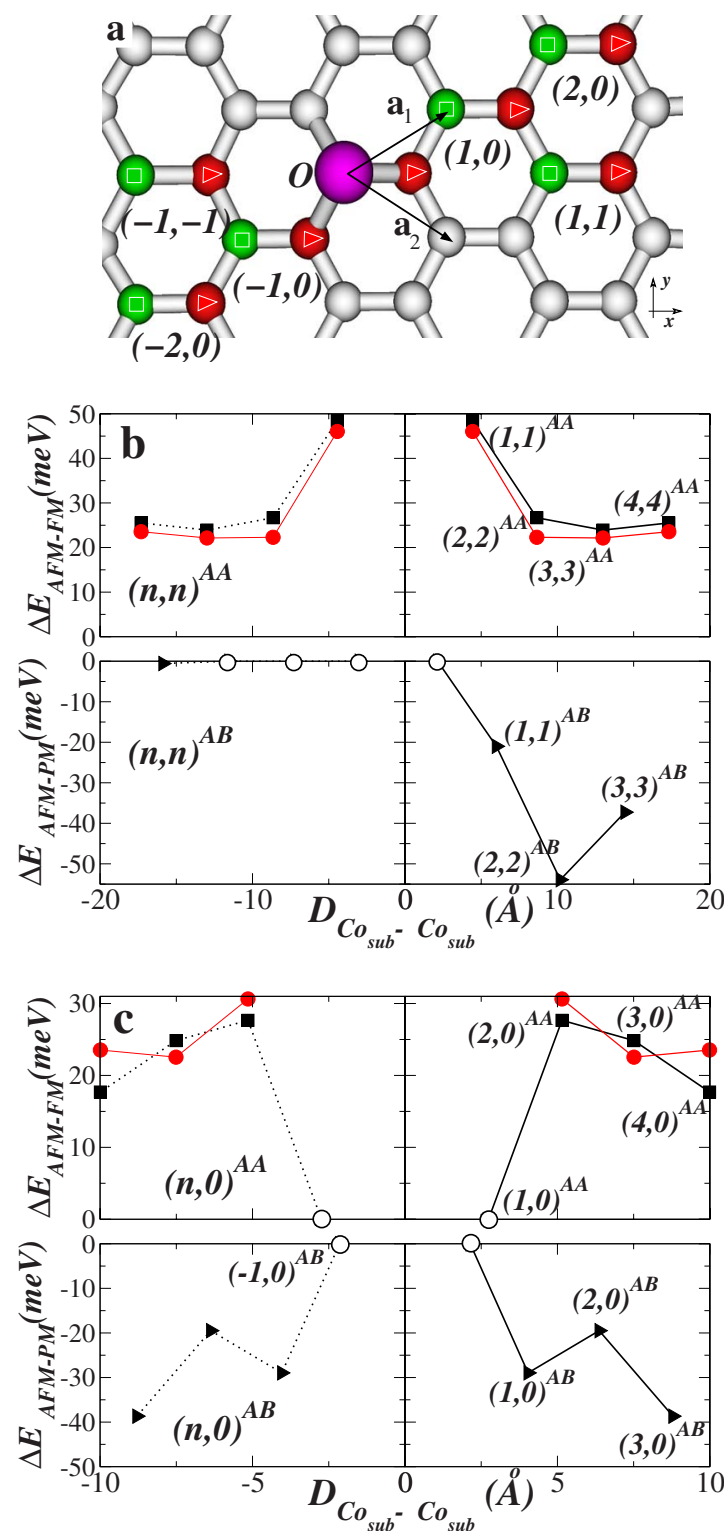

FIG. 3. (Color online) (a) Schematic representation of the geometry used to calculate the relative stability of FM, AFM, and PM solutions as a function of the positions of two $\mathrm{Co}_{s u b}$ impurities. Sublattices $A$ and $B$ are indicated by squares and triangles, respectively. One of the impurities is fixed in a central $A$-type site, whereas the other is moved along the (b) $(n, n)$ and (c) $(n, 0)$ directions. The empty circles represent spin compensated solutions and the full circles correspond to a fit with a Heisenberg model (see text). Positive values indicate FM spin alignment while negative values are ones.

alignments as a function of the relative position of the defects. Figure 3 shows the results along with a schematic representation of our notation. These energies are comparable to the spin-flip energies in ferromagnetic transition metals $\mathrm{Fe}$, $\mathrm{Co}$, and Ni. Positive values indicate ferromagnetic (FM) spin alignment while negative values are antiferromagnetic (AFM) ones. Several observations from spin couplings in Fig. 3 can be made: (i) when the impurities are located in the same sublattice ( $A A$ systems) the FM configuration is more stable than the AFM one; (ii) if the Co atoms are in opposite 


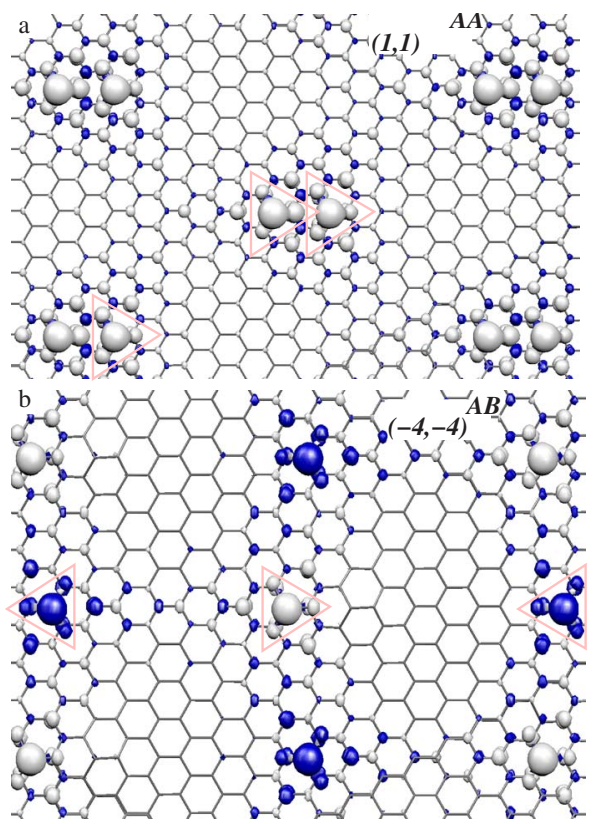

FIG. 4. (Color online) (a) Spin densities for configurations (a) $(1,1)^{A A}$, and (b) $(-4,-4)^{A B}$ [see Fig. 3(a) for the nomenclature]. Positive and negative spin densities are indicated by light (gray) and

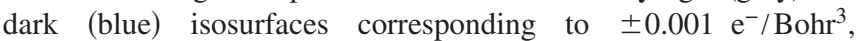
respectively.

sublattices ( $A B$ systems) it is very difficult to reach a FM solution, ${ }^{27}$ instead the system finds either a spin compensated (PM) or an AFM solution; (iii) at short distances $(<3.0 \AA)$ the systems always converge to spin compensated solutions.

In the FM cases of Fig. 3, the total spin magnetization integrates to $2.00 \mu_{B}$, with a spin population on every Co atom that remains almost constant $\sim 0.50 \mu_{B}\left(\sim 0.30 \mu_{B}\right.$ for the three $\mathrm{C}$ nearest neighbors). In other cases the total spin is zero. Thus, the total magnetic moment of the system follows the equation $S=\left|\mathrm{N}_{\text {sub }}^{A}-\mathrm{N}_{\text {sub }}^{B}\right|$, where $\mathrm{N}_{\text {sub }}^{A(B)}$ is the number of $\mathrm{Co}_{\text {sub }}$ defects in the $A(B)$ sublattices. Therefore, our total moment is consistent with Lieb's theorem for bipartite lattices. ${ }^{16}$ This supports our analogy between the electronic structure of $\mathrm{Co}_{\text {sub }}$ defects and single vacancies in a simplified $\pi$ tight-binding description of graphene.

The spin magnetization density for some selected configurations is plotted in Fig. 4. Although the spin is fairly localized on the Co atom and the neighboring $\mathrm{C}$ atoms, the presence of the defect also causes a delocalized magnetization density with alternated signs in the two sublattices. The triangular pattern, that reflects the threefold symmetry of the layer, shows different orientations for $A$ and $B$ substitutions. This explains the very anisotropic $A B$ interaction along the $(n, n)$ direction seen in Fig. 3(b): the energy difference between AFM and PM solutions for $(n, n)^{A B}$ configurations strongly depends on the relative position of the impurities, showing such directionality. Similar patterns have already been observed experimentally ${ }^{28-31}$ in point defects (e.g., vacancies and hydrogens) on graphene by means of STM techniques and also theoretically discussed for $\pi$ vacancies. ${ }^{8,11,32}$ For $\mathrm{Co}_{\text {sub }}$ in graphene, similar STM experiments should display the topology of the spin densities given in Fig. 4.
We have also investigated the magnetic interactions within the framework of a classical Heisenberg model, $H$ $=\sum_{i<j} J_{A A / A B}\left(\mathbf{r}_{i j}\right) \mathbf{S}_{i} \mathbf{S}_{j}$, where $\mathbf{S}_{i}$ is the local moment for a $\mathrm{Co}_{\text {sub }}$ impurity at site $i$. The expression for the $\mathbf{r}_{i j}$ dependence of the exchange has been taken from analytical RKKY coupling given in Ref. 33, except for the exponent of the distance decay, which is fitted to our ab initio results. The exchange interaction for $A A$ systems can be fitted with a $\left|r_{i j}\right|^{-2.43}$ distance dependence [see the full circles in Figs. 3(b) and 3(c)]. This distance dependence is in reasonable agreement with the $\left|r_{i j}\right|^{-3}$ behavior obtained with analytical models for substitutional defects and voids. ${ }^{33,34}$ In the case of $A B$ systems a simple RKKY-like treatment fails to satisfactorily describe the interactions, at least for the relatively short distances between defects considered in our calculations.

Next, we explain the appearance of PM solutions in Fig. 3. The appreciable interaction between defect levels in neighboring impurities for $A B$ systems opens a bondingantibonding gap in the $p_{z}$ defect band ${ }^{35}$ and, thus, contributes to the stabilization of PM solutions. For $A A$ systems, however, the bipartite character of the graphene lattice makes the interaction between defects much smaller. This explains the prevalence of solutions showing a local spin polarization for $A A$ configurations. For very short distance between impurities, a larger defect-defect interaction opens a large gap and, in consequence, stabilizes PM configurations. It is interesting to point out that similar behaviors were previously observed for vacancies in graphene and graphene ribbons described within a simplified $\pi$ tight-binding model. ${ }^{8,32,36}$

In this section, we dealt with the spin and magnetic coupling for several $\mathrm{Co}_{\text {sub }}$ atoms in a $8 \times 8$ supercell. As shown in Fig. 1(a), it is easy to visualize the electronic spin-density distribution of the defect level band induced by a Co substitutional. The spin density shows maxima in the opposite $\mathrm{C}$ atoms of the Co substitutional site, reflecting the bipartite character of graphene lattice. For two Co substitutionals, we distinguish between $A A$ and $A B$ positions. For $A A$ cases, the band induced by Co impurities remains close to the Fermi level. This indicates a small hopping between $A$ sites. Similar to a Co substitutional, these band origins that the spin moment becomes $1 \mu_{B}$ per Co atom. Additionally, we observe that $A A$ moments align ferromagnetically and depend on distance as RKKY-like interactions. Hence, the total moment of AA follow Lieb's theorem similarly to a Co substitutional per cell. For $\mathrm{AB}$ cases, the band structures around Fermi energy show the opening of antibonding-bonding gaps and suggest large hoppings between these sites. The alignments of the magnetic moments are AFM or PM, trend that is similar to $\pi$ vacancies. ${ }^{8,32,36}$ To end this section we ought to remark that the origin of the spin moment and sign couplings stems for the bipartite lattice of graphene.

\section{Link with experiments}

Similar behaviors to $\mathrm{Co}_{s u b}$ are expected for any other system that can be mapped into a simple $\pi$-vacancy in graphene. This is the case of a hydrogen atom saturating a C $p_{z}$ state in graphene. ${ }^{37,38}$ However, there are two important differences between these two systems. As Co prefers ener- 
getically to sit on carbon vacancies, $\mathrm{Co}_{\text {sub }}$ defects could be fabricated by depositing Co atoms on areas where vacancies have been previously created in graphenic nanostructures by, for example, electron irradiation. ${ }^{39}$ The calculated diffusion barrier of hydrogen on graphene is $1.19 \mathrm{eV},{ }^{40}$ the experimentally observed activation energy for the migration of substitutional metals such as $\mathrm{Au}$ and $\mathrm{Pt}$, in graphene is around 2.5 eV. ${ }^{14}$ Thus, the diffusion is also probably much slower for $\mathrm{Co}_{\text {sub }}$ defects than for hydrogen atoms. We expect that stable arrays of $\mathrm{Co}_{\text {sub }}$ impurities could be fabricated and open a route to obtain in reality many of the interesting magnetic behaviors obtained for $\pi$ models of defective graphene.

\section{CONCLUSIONS}

In summary, only Co substitutional atoms in graphene respect to other traditional ferromagnetic materials such as $\mathrm{Fe}$ and $\mathrm{Ni}$, present spin polarization. Thus, we focus on Co substitutionals and analyze their magnetic couplings in the $A A$ and $A B$ sites of graphene lattice. The electronic structure of
$\mathrm{Co}_{\text {sub }}$ defects (see Fig. 1) is dominated by an impurity level at $\mathrm{E}_{F}$ with strong contribution from the $p_{z}$ states of the neighboring $\mathrm{C}$ atoms, and it is a clear reminiscent of a single vacancy in a simplified $\pi$ tight-binding description of graphene. The study of the Co coupling in several sites demonstrates FM alignments for the $A A$ cases, in contradistinction to $A B$ cases which are either AFM or spin compensated. The $A A$ cases were fitted to a Heisenberg model with a RKKY exchange constant. Our findings for $\mathrm{Co}_{s u b}$ can be used to bring into contact the engineering of nanostructures with the results of $\pi$ models in defective graphene. Further works could be done to investigate the magnetic couplings for intrinsic defects such as double vacancies ${ }^{41}$ or adatoms in graphene.

\section{ACKNOWLEDGMENTS}

We acknowledge support from Basque Dep. de Educación and the UPV/EHU (Grant No. IT-366-07) and the Spanish MCI (Grant No. FIS2007-66711-C02-02).

\footnotetext{
*eltonjose_gomes@ehu.es

†sqbsapod@ehu.es

*Corresponding author; swxayfea@sw.ehu.es

${ }^{1}$ K. S. Novoselov, A. K. Geim, S. V. Morozov, D. Jiang, Y. Zhang, S. V. Dubonos, I. V. Grigorieva, and A. A. Firsov Science 306, 666 (2004).

${ }^{2}$ K. S. Novoselov, A. K. Geim, S. V. Morozov, D. Jiang, M. I. Katsnelson, I. V. Grigorieva, S. V. Dubonos, and A. A. Firsov, Nature (London) 438, 197 (2005).

${ }^{3}$ A. K. Geim and K. S. Novoselov, Nature Mater. 6, 183 (2007).

${ }^{4}$ A. H. Castro Neto, F. Guinea, N. M. R. Peres, K. S. Novoselov, and A. K. Geim, Rev. Mod. Phys. 81, 109 (2009).

${ }^{5}$ Carbon Based Magnetism, edited by T. Makarova and F. Palacios (Elsevier, Amsterdam, 2006).

${ }^{6}$ H. Amara, S. Latil, V. Meunier, P. Lambin, and J. C. Charlier, Phys. Rev. B 76, 115423 (2007).

${ }^{7}$ P. O. Lehtinen, A. S. Foster, A. Ayuela, A. Krasheninnikov, K. Nordlund, and R. M. Nieminen, Phys. Rev. Lett. 91, 017202 (2003).

${ }^{8}$ J. J. Palacios, J. Fernández-Rossier, and L. Brey, Phys. Rev. B 77, 195428 (2008)

${ }^{9}$ A. V. Krasheninnikov, P. O. Lehtinen, A. S. Foster, P. Pyykkö, and R. M. Nieminen, Phys. Rev. Lett. 102, 126807 (2009).

${ }^{10}$ N. M. R. Peres, F. D. Klironomos, S.-W. Tsai, J. R. Santos, J. M. B. Lopes dos Santos, and A. H. Castro Neto, Europhys. Lett. 80, 67007 (2007).

${ }^{11}$ V. M. Pereira, J. M. B. Lopes dos Santos, and A. H. Castro Neto, Phys. Rev. B 77, 115109 (2008).

${ }^{12}$ T. O. Wehling, A. V. Balatsky, M. I. Katsnelson, A. I. Lichtenstein, K. Scharnberg, and R. Wiesendanger, Phys. Rev. B 75, 125425 (2007).

${ }^{13}$ E. J. G. Santos, A. Ayuela, S. B. Fagan, J. Mendes Filho, D. L. Azevedo, A. G. Souza Filho, and D. Sanchez-Portal, Phys. Rev. B 78, 195420 (2008).

${ }^{14}$ Y. Gan, L. Sun, and F. Banhart, Small 4, 587 (2008).
}

${ }^{15}$ E. Santos, A. Ayuela, and D. Sanchez-Portal, arXiv:0910.0400 (unpublished).

${ }^{16}$ E. H. Lieb, Phys. Rev. Lett. 62, 1201 (1989).

${ }^{17}$ D. Sánchez-Portal, E. Artacho, and J. M. Soler, Int. J. Quantum Chem. 65, 453 (1997).

${ }^{18}$ J. M. Soler, Emilio Artacho, Julian D. Gale, Alberto García, Javier Junquera, Pablo Ordejón, and Daniel Sánchez-Portal, J. Phys.: Condens. Matter 14, 2745 (2002).

${ }^{19}$ N. Troullier and J. L. Martins, Phys. Rev. B 43, 1993 (1991).

${ }^{20}$ S. G. Louie, S. Froyen, and M. L. Cohen, Phys. Rev. B 26, 1738 (1982).

${ }^{21}$ G. Kresse and J. Hafner, Phys. Rev. B 47, 558 (1993).

${ }^{22}$ G. Kresse and J. Furthmüller, Phys. Rev. B 54, 11169 (1996).

${ }^{23}$ M. Ushiro, K. Uno, T. Fujikawa, Y. Sato, K. Tohji, F. Watari, W. J. Chun, Y. Koike, and K. Asakura, Phys. Rev. B 73, 144103 (2006).

${ }^{24}$ D. Kitchen, Anthony Richardella, Jian-Ming Tang, Michael E. Flatté, and Ali Yazdani, Nature (London) 442, 436 (2006).

${ }^{25}$ Only Co has a partially occupied band, related to the impurity, at the Fermi energy composed mostly by $p_{z} \mathrm{C}$ character. In contrast, the band character at Fermi energy for $\mathrm{Fe}$ is mainly $3 d$ and $\mathrm{Ni}$ substitutionals is mainly $s p \mathrm{C}$ from the $\mathrm{C}$ neighbors.

${ }^{26}$ M. Khantha, N. A. Cordero, L. M. Molina, J. A. Alonso, and L. A. Girifalco, Phys. Rev. B 70, 125422 (2004); Y. SanchezPaisal, D. Sanchez-Portal, and A. Ayuela, ibid. 80, 045428 (2009); Y. Sanchez-Paisal, D. Sanchez-Portal, N. Garmendia, R. Muñoz, I. Obieta, J. Arbiol, L. Calvo-Barrio, and A. Ayuela, Appl. Phys. Lett. 93, 053101 (2008).

${ }^{27}$ When we could stabilize a FM solution, it lies at higher energy, around $0.2 \mathrm{eV}$ above the PM one.

${ }^{28}$ K. F. Kelly and N. J. Halas, Surf. Sci. 416, L1085 (1998).

${ }^{29}$ H. A. Mizes and J. S. Foster, Science 244, 559 (1989).

${ }^{30}$ G. M. Rutter, J. N. Crain, N. P. Guisinger, T. Li, P. N. First, and J. A. Stroscio, Science 317, 219 (2007).

${ }^{31}$ P. Ruffieux, O. Groning, P. Schwaller, L. Schlapbach, and 
P. Groning, Phys. Rev. Lett. 84, 4910 (2000).

${ }^{32}$ O. V. Yazyev, Phys. Rev. Lett. 101, 037203 (2008).

${ }^{33}$ S. Saremi, Phys. Rev. B 76, 184430 (2007).

${ }^{34}$ M. A. H. Vozmediano, M. P. Lopez-Sancho, T. Stauber, and F. Guinea, Phys. Rev. B 72, 155121 (2005).

${ }^{35}$ For the $A B$ systems, we always find bonding-antibonding gaps in the defect band ranging from 0.3 to $0.9 \mathrm{eV}$ when going from $(1,1)^{A B}$ to the $(-1,-1)^{A B}$ configuration, respectively. These values have to be compared with the $\sim 0.5 \mathrm{eV}$ spin splitting of the $\mathrm{Co}_{\text {sub }}$ defect. All those $A B$ systems with gaps larger than $0.4 \mathrm{eV}$ are found to converge to PM solutions.
${ }^{36}$ H. Kumazaki and D. S. Hirashima, J. Phys. Soc. Jpn. 76, 064713 (2007).

${ }^{37}$ O. V. Yazyev and L. Helm, Phys. Rev. B 75, 125408 (2007).

${ }^{38}$ D. W. Boukhvalov, M. I. Katsnelson, and A. I. Lichtenstein, Phys. Rev. B 77, 035427 (2008).

${ }^{39}$ J. A. Rodriguez-Manzo and F. Banhart, Nano Lett. 9, 2285 (2009).

${ }^{40}$ Yang Lei, S. A. Shevlin, W. Zhu, and Z. X. Guo, Phys. Rev. B 77, 134114 (2008).

${ }^{41}$ D. W. Boukhvalov and M. I. Katsnelson, Appl. Phys. Lett. 95, 023109 (2009). 